Revisión

\title{
Resultados e impacto de la investigación en genética y mejoramiento genético de las abejas melíferas desarrollada por el INIFAP en México
}

\author{
Miguel Enrique Arechavaleta-Velasco ${ }^{\text {a* }}$ \\ Claudia García-Figueroa ${ }^{a}$ \\ Laura Yavarik Alvarado-Avila ${ }^{\text {a }}$ \\ Francisco Javier Ramírez-Ramírez ${ }^{\text {a }}$ \\ Karla Itzel Alcalá-Escamilla ${ }^{\text {a }}$
}

${ }^{a}$ Instituto Nacional de Investigaciones Forestales, Agrícolas y Pecuarias. Centro Nacional de Investigación Disciplinaria en Fisiología y Mejoramiento Animal. km 1 Carretera a Colón; Ajuchitlán, Colón, Querétaro. México.

*Autor de correspondencia: arechavaleta.miguel@inifap.gob.mx

\section{Resumen:}

La apicultura es una actividad que tiene importancia económica, social y ecológica. En México esta actividad enfrenta varios problemas, entre los que destacan el alto comportamiento defensivo de las colonias de abejas melíferas (Apis mellifera L.) provocado por la africanización y la varroosis ocasionada por el ácaro Varroa destructor. El alto comportamiento defensivo de las colonias ha ocasionado que la apicultura sea más compleja y menos rentable, mientras que la varroosis afecta la producción de miel de las colonias, y es un factor asociado con la pérdida de colonias de abejas que ocurre a nivel mundial. Para atender estos problemas el INIFAP desarrolla investigación en genética y mejoramiento genético apícola. El objetivo de este artículo fue hacer una revisión de los resultados de los trabajos de investigación en genética en los que el INIFAP ha estado involucrado que han permitido generar conocimientos científicos sobre los factores genéticos, genómicos y epigenéticos que regulan la expresión de los comportamientos defensivo, de guardia, de aguijoneo, de acicalamiento e higiénico de las abejas. De los trabajos desarrollados en materia de mejoramiento genético para reducir el comportamiento defensivo de las abejas que han permitido generar métodos para la evaluación y selección de esta característica, así como generar líneas de abejas de bajo 
comportamiento defensivo a partir de las cuales se han transferido reinas a los productores. Así como de los trabajos para la conservación de material genético de origen europeo que derivaron en el establecimiento de un Banco de Germoplasma Apícola.

Palabras clave: Abejas melíferas, Mejoramiento Genético, Comportamiento defensivo, Varroa destructor, Comportamiento de acicalamiento.

Recibido: 04/01/2021

Aceptado: 11/03/2021

\section{Introducción}

\section{Importancia de la apicultura en México}

México es uno de los principales productores y exportadores de miel de abeja en el mundo, y actualmente se ubica como el décimo productor de miel y el quinto exportador a nivel mundial ${ }^{(1)}$. Durante el periodo comprendido entre 2010 y 2019, la producción anual de miel fue de 58,094 t en promedio, con un rendimiento anual promedio de 29.7 $\mathrm{kg}$ por colmena ${ }^{(2)}$. En este mismo periodo se exportaron anualmente en promedio 34,745 t de miel lo que representa el $60 \%$ de la producción ${ }^{(1)}$, ya que la miel que se produce en México posee características de calidad que hace que tenga alta demanda en el mercado internacional. El valor comercial de la producción de miel en México para el periodo 2010-2019 fue de 2,924 millones de pesos anuales en promedio ${ }^{(1)}$.

En México existen 2,157,866 colonias de abejas que son propiedad de aproximadamente 43,000 apicultores $^{(2)}$. La apicultura es una actividad socialmente importante porque aproximadamente el $70 \%$ de los apicultores son pequeños productores, para quienes la venta de miel y cera representa una parte importante de sus ingresos. Se estima que el $60 \%$ de las colonias que existen en México son propiedad de este tipo de apicultores, quienes tienen un bajo grado de tecnificación y manejan 40 colmenas en promedio, el resto de las colonias (40\%) son propiedad de unidades de producción apícola de diversos tamaños y grados de tecnificación ${ }^{(3)}$.

Además de la producción de miel, cera, polen, jalea real y propóleo, la polinización que realizan las abejas en las plantas es muy importante para el equilibrio de los ecosistemas y para la producción de alimentos. Se estima que el $70 \%$ de las plantas cultivadas que son utilizadas para consumo humano son polinizadas en forma parcial o total por las abejas melíferas, por lo que la alimentación del ser humano depende en forma importante de alimentos en los que las abejas participaron para su producción. Se calcula que el valor de la polinización que realizan las abejas en plantas cultivadas en México es 20 veces el valor de lo generado por la producción de $\operatorname{miel}^{(4)}$, por lo que el valor estimado para la 
polinización que realizaron las abejas en plantas cultivadas en el periodo comprendido entre 2010 y 2019 fue de 58,480 millones de pesos por año.

México se divide en cinco regiones apícolas: Norte, Altiplano, Costa del Pacífico, Golfo y Península de Yucatán. Estas regiones están determinadas con base en el tipo de clima y el tipo de vegetación predominante ${ }^{(5)}$. Las regiones Península de Yucatán, Altiplano y Costa del Pacífico son las que contribuyen con la mayor parte de la producción de miel. En la península de Yucatán se produce el $36 \%$ del total de la producción de miel nacional, lo que equivale al $32 \%$ del valor económico de la producción; mientras que en el Altiplano se produce el $25 \%$ de la miel nacional y representa el $27 \%$ del valor económico de la producción, la región Costa del Pacífico produce el $22 \%$ y contribuye con el $24 \%$ del valor económico total, mientras que en la región Norte se produce el $9 \%$ de la miel de nuestro país y representa el $9 \%$ del valor económico total, y la región del Golfo produce el $8 \%$ de la miel nacional que corresponde al $8 \%$ del valor económico de la producción $^{(2)}$.

\section{Principales problemas de la apicultura en México en los que el INIFAP ha realizado investigación}

El alto comportamiento defensivo de las colonias de abejas ocasionado por la africanización de las poblaciones de abejas y la varroosis ocasionada por el ácaro Varroa destructor, son los dos principales problemas que enfrenta la apicultura en México, para ambos el INIFAP ha desarrollado investigación en las áreas de genética y mejoramiento con el objeto de contribuir a reducir su impacto.

El proceso de africanización de las poblaciones de abejas que ha ocurrido durante los últimos 60 años en el continente americano es considerado como uno de los casos de mayor impacto de una especie invasiva en la historia. Las abejas africanizadas se originaron en Brasil al cruzarse abejas de origen europeo (A. mellifera mellifera, A. mellifera ligustica y A. mellifera carnica) con abejas de origen africano (A. mellifera scutellata) que fueron introducidas en ese país en $1956^{(6)}$.

Las abejas africanizadas tienen etapas de desarrollo de la cría $\operatorname{cortas}^{(7)}$ y tasas reproductivas altas que se traducen en una alta producción de enjambres, lo que facilitó su dispersión a través de Sudamérica y Centroamérica, llegando a México en $1986^{(8)}$ y a Estados Unidos de América en $1990^{(9)}$.

La africanización tuvo consecuencias drásticas para la apicultura e incluso para la salud pública de muchos países ${ }^{(10)}$. La apicultura en México ha sufrido cambios importantes debido a la africanización de las poblaciones de abejas desde que la abeja africanizada llegó al país en $1986^{(11)}$, ya que estas abejas presentan un mayor comportamiento defensivo, una mayor tendencia a enjambrar y a evadir, y algunos estudios indican que producen menos miel para el apicultor que las abejas europeas ${ }^{(12-15)}$. 
El alto comportamiento defensivo de las colonias de abejas ocasionado por la africanización ha hecho que el manejo de las colonias sea más complejo, lo que a su vez ha provocado que la actividad sea menos rentable debido al aumento en los costos de producción asociado a las prácticas que los apicultores han tenido que implementar para el manejo de este tipo de $\operatorname{abejas}^{(11)}$.

Las abejas africanizadas se encuentran en todo el país y el grado de africanización de las poblaciones de abejas que manejan los apicultores es alto ${ }^{(16)}$. Un estudio que se realizó en Yucatán indicó que el $61 \%$ de las colonias bajo manejo y el 87 \%de las colonias silvestres analizadas eran africanizadas ${ }^{(17)}$, mientras que otro estudio posterior indica que el $100 \%$ de las colonias analizadas fueron africanizadas ${ }^{(16)}$. Un estudio que se realizó en el Estado de México estimó que el $37 \%$ de las colonias que manejan los apicultores en la entidad eran africanizadas y el $70 \%$ tenía algún grado de africanización ${ }^{(18)}$. Mientras que un estudio que se llevó a cabo en Morelos estimó que el $65 \%$ de las colonias tenían algún grado de africanización ${ }^{(19)}$.

La varroosis representa una seria amenaza para la supervivencia de las abejas melíferas y para la producción de miel tanto en México como en el mundo. Un estudio que se realizó en México indica que colonias con niveles de infestación de Varroa destructor del $6 \%$ produjeron en promedio $65 \%$ menos miel que colonias libres del parásito ${ }^{(20)}$, mientras que otro estudio reporta que la producción de miel de las colonias se redujo en $52.8 \mathrm{~g}$ por cada unidad de porcentaje de infestación de este ácaro ${ }^{(21)}$. Asimismo, este ácaro ha sido asociado a la presencia del síndrome del colapso de las colonias (CCD), fenómeno responsable de la pérdida de colonias de abejas que ha ocurrido en México ${ }^{(22,23)}$ y en otras partes del mundo en los últimos años ${ }^{(24-27)}$.

Actualmente, la varroosis se controla utilizando acaricidas químicos de síntesis y productos químicos orgánicos. Ninguno de los productos tiene una eficacia del $100 \%$ para el control del ácaro. Los acaricidas de síntesis que más se utilizan en México son la flumetrina y el fluvalinato que reportan una eficacia del $98 \%$; sin embargo, existen reportes tanto en México como en otras partes del mundo de poblaciones de ácaros que han generado resistencia a estos productos, en donde los acaricidas tienen una eficacia para el control de $V$. destructor menor al $50 \%{ }^{(28,29)}$. Además, el uso de estos productos puede dejar residuos químicos en la miel y cera que afectan tanto a las abejas como a los humanos ${ }^{(30-34)}$. Los productos químicos orgánicos que más se utilizan en México para el control del ácaro son el timol, el ácido oxálico y el ácido fórmico; si bien estos productos no generan resistencia en las poblaciones de ácaros, la eficacia para el control del parásito de estos productos es de $93 \%$ o enos $^{(35)}$.

Varroa destructor fue detectado por primera vez en México en $1992^{(36)}$ y actualmente se encuentra distribuido en todo el país. Un estudio que se llevó a cabo en Zacatecas reportó que la prevalencia de la varroosis es de $89 \%$ y el nivel de infestación promedio de Varroa destructor es de $4.85 \%{ }^{(37)}$, en Morelos se reportó una prevalencia del $80 \%$ y un nivel de infestación promedio de $4.76 \%{ }^{(38)}$, en Jalisco se reportó una prevalencia de $88 \%$ y un 
nivel de infestación de $5.2 \%{ }^{(39)}$, en Yucatán se reportó una prevalencia de $63 \%$ y un nivel de infestación de $1.70 \%{ }^{(40)}$.

El objetivo de este artículo es hacer una revisión de los resultados de los trabajos de investigación desarrollados en México por el Instituto Nacional de Investigaciones Forestales, Agrícolas y Pecuarias, así como de trabajos desarrollados fuera del país en los que participaron investigadores del INIFAP en materia de genética y mejoramiento genético de las abejas relacionados con el comportamiento defensivo de las abejas, y la resistencia de las abejas a la varroosis.

\section{Resultados de estudios realizados sobre la genética del comportamiento defensivo}

Los estudios que se han llevado a cabo en México con objeto de conocer los mecanismos genéticos que regulan la expresión del comportamiento defensivo de las abejas han permitido determinar que el comportamiento defensivo a nivel de la colonia, medido por el número de aguijones que las abejas de una colonia depositan sobre una bandera de gamuza negra que se agita frente a la colmena por un tiempo determinado, está regulado por efectos genéticos de dominancia, ya que al comparar esta característica entre los grupos genéticos europeo, africanizado e híbrido, se encontró que existen diferencias significativas en el comportamiento defensivo de los grupos europeos y africanizados, y que el comportamiento defensivo de las colonias del grupo híbrido es tan alto como el del grupo africanizado. Asimismo, estos estudios permitieron determinar que las interacciones entre abejas de los tres grupos genéticos dentro de una colonia influyen en el comportamiento defensivo de la colonia de abejas, es decir que si en una colonia coexisten abejas con genotipo europeo y abejas con genotipo africano o híbrido, el comportamiento defensivo de esa colonia será tan alto como el de una colonia formada únicamente por abejas híbridas o africanizadas ${ }^{(41,42,43)}$.

En México se llevó a cabo el primer estudio para identificar las regiones dentro del genoma de la abeja que regulan la expresión del comportamiento defensivo de las colonias. En este estudio, se identificaron cinco Loci de Rasgos Cuantitativos o QTL (por sus siglas en inglés) ligados a la expresión de esta característica, utilizando una población de 172 colonias formadas por una sola familia de obreras retrocruzadas que se generaron utilizando un esquema de cruzamientos a partir de abejas africanizadas y europeas ${ }^{(14)}$. El efecto sobre el comportamiento defensivo de las abejas de tres de los cinco QTL que fueron identificados en este estudio, se confirmó posteriormente en dos poblaciones independientes, tanto de abejas africanizadas ${ }^{(43)}$, como de abejas europeas ${ }^{(44)}$.

La respuesta defensiva de una colonia involucra dos comportamientos que realizan las obreras de la colonia a nivel individual, el comportamiento de guardia y el comportamiento de aguijoneo ${ }^{(45-51)}$. En México se realizaron algunos estudios que permitieron comprender cómo se relacionan el comportamiento de guardia y el 
comportamiento de aguijoneo durante la respuesta defensiva de una colonia de abejas; los resultados indican que las abejas que realizan el comportamiento de guardia, participan en la respuesta defensiva de una colonia de tal forma que su presencia, su número y la proporción de ellas que reaccionan picando, influye en la intensidad de la respuesta defensiva de la colonia ${ }^{(44,50,51)}$.

Asimismo, en México se han realizado estudios para conocer los mecanismos genéticos que regulan la expresión del comportamiento de guardia y el comportamiento de aguijoneo a nivel de abejas individuales, lo cual ha sido importante para entender cómo es que comportamientos que son regulados genéticamente a nivel de los individuos influyen sobre el fenotipo de toda la colonia.

En el caso del comportamiento de aguijoneo, los estudios que se han realizado permitieron identificar que a nivel de abejas individuales el comportamiento de aguijoneo (medido como el tiempo de reacción que tarda una abeja en aguijonear un pedazo de gamuza negra al aplicarle un estímulo eléctrico constante), fue significativamente mayor en las abejas africanizadas que en las abejas europeas, es decir, las abejas africanizadas respondieron aguijoneando en menos tiempo que las abejas europeas. Asimismo, en uno de estos estudios se encontró que el comportamiento de aguijoneo de abejas que realizan funciones de guardia en la entrada de la colmena y el de abejas que responden intentando aguijonear a un intruso, fue superior al de abejas que realizan otras labores dentro de la colonia, tanto en colonias de origen europeo como africanizado ${ }^{(49)}$.

En cuanto a la identificación de regiones genómicas que regulan la expresión del comportamiento de aguijoneo a nivel de abejas individuales, el estudio desarrollado por Arechavaleta-Velasco et al ${ }^{(44)}$ determinó que tres de los QTL que habían sido previamente asociados con el comportamiento defensivo de las abejas a nivel de toda la colonia ${ }^{(14)}$ tienen efecto sobre el comportamiento de aguijoneo de abejas individuales, mientras que en el estudio desarrollado por Shorter et $a l^{(52)}$ se identificaron dos nuevos QTL para esta característica, a partir de los cuales se pudieron identificar cuatro genes candidatos para la expresión del comportamiento de aguijoneo de las abejas.

En el caso del comportamiento de guardia, los estudios que se han realizado permitieron identificar que existen efectos de origen genético para el número de abejas que realizan el comportamiento de guardia en una colonia de abejas ${ }^{(44)}$.

El estudio desarrollado por Arechavaleta-Velasco y Hunt ${ }^{(53)}$ para identificar las regiones en el genoma que regulan la expresión del comportamiento de guardia en abejas obreras, permitió detectar siete loci de Rasgos Binarios o BTL (por sus siglas en inglés) asociados a la expresión de esta característica, utilizando dos colonias de abejas producto de retrocruzas recíprocas generadas a partir de líneas de abejas europeas de alto y bajo comportamiento defensivo. Asimismo, en otro estudio se determinó que uno de los QTL que habían sido previamente asociados con el comportamiento defensivo de las abejas a nivel de toda la colonia ${ }^{(14)}$, tiene efecto sobre el comportamiento de guardia de abejas 
individuales ${ }^{(44)}$, mientras que en el estudio desarrollado por Shorter et a $l^{(52)}$ se identificó un nuevo QTL para esta característica, y a partir de éste se pudieron identificar tres genes candidatos para la expresión del comportamiento de guardia de las abejas.

Guzmán-Novoa et $a l^{(54)}$ identificaron que el comportamiento defensivo de colonias formadas por abejas de los dos genotipos híbridos generados a partir de cruzas recíprocas entre abejas africanizadas y europeas es diferente; las colonias híbridas producto de la cruza entre reinas europeas y zánganos africanizados son significativamente más defensivas que las colonias híbridas producto de la cruza entre reinas africanizadas y zánganos europeos. Las diferencias fenotípicas entre grupos híbridos recíprocos es un indicador de la presencia de efectos epigenéticos ${ }^{(55,56)}$, por lo que estos resultados sugieren que el mayor comportamiento defensivo de las colonias cuyo origen paterno es africanizado se debe a efectos epigenéticos generados por un proceso de impronta de origen paterno.

En el estudio desarrollado por Kocher et $a l^{(57)}$ se analizó el transcriptoma de diferentes tejidos de abejas de los genotipos híbridos recíprocos generados a partir de un esquema de cruzamientos entre abejas africanizadas y europeas, y se encontró que la expresión selectiva de genes por su origen parental es uno de los mecanismos mediante los cuales se presentan efectos epigenéticos en las abejas. La expresión selectiva de genes por su origen parental ocurre cuando el nivel de expresión de un gen depende de si un alelo se hereda por vía materna o paterna.

Gibson et $a l^{(58)}$ encontraron que para el comportamiento de aguijoneo de abejas individuales existen diferencias entre los genotipos híbridos recíprocos que provienen de cruzas entre abejas europeas y africanizadas. Las abejas híbridas cuyo origen paterno era africanizado respondieron aguijoneando un pedazo de gamuza negra al aplicarles un estímulo eléctrico significativamente más rápido que las abejas híbridas cuyo origen paterno era europeo, lo que, al igual que para el comportamiento defensivo a nivel de toda la colonia de abejas, es indicador de la presencia de efectos epigenéticos por un proceso de impronta de origen paterno para esta característica.

Para determinar si los efectos epigenéticos detectados en el comportamiento de aguijoneo de abejas individuales ${ }^{(58)}$ se deben a la expresión selectiva de genes por su origen parental $^{(59)}$, se realizó un análisis del transcriptoma de genes localizados en las regiones genómicas que corresponden a los QTL asociados al comportamiento defensivo que fueron detectados en estudios previos ${ }^{(14,43,44,52)}$; este análisis se realizó en las abejas a las que se les evaluó el comportamiento de aguijoneo en forma individual, y se encontró que este mecanismo está involucrado en la regulación de la expresión de estos genes en las abejas híbridas cuyo origen materno es europeo, y cuyo origen paterno es africanizado, aparentemente por perturbaciones en las rutas de señalización establecidas entre los genes nucleares y mitocondriales que modulan el metabolismo del cerebro y el comportamiento defensivo en las abejas ${ }^{(58)}$. 


\section{Resultados de estudios y programas de mejoramiento genético para reducir el comportamiento defensivo de las abejas}

El estudio publicado por Guzmán-Novoa y Page ${ }^{(59)}$ en donde se reportan los resultados de un programa de selección que inició en noviembre de 1991 y que concluyó en 1996, que se implementó durante cuatro generaciones de selección en una población comercial de aproximadamente 3,000 colonias de abejas, con el objetivo de mejorar la producción de miel y reducir el comportamiento defensivo de la población, demostró que es posible hacer mejoramiento genético de las poblaciones de abejas en zonas africanizadas, sin utilizar inseminación instrumental de reinas.

El programa de mejoramiento genético consistió en realizar selección masal por etapas, considerando las características de producción de miel, comportamiento defensivo, patrón de postura de las reinas y longitud promedio del ala anterior de las abejas obreras de la colonia. Los resultados del programa mostraron que fue posible mantener la producción de miel, reducir el comportamiento defensivo de las colonias e incrementar la longitud promedio del ala anterior de las abejas obreras en la población bajo selección, pese al proceso de africanización de las abejas que estaba ocurriendo en ese momento en la región donde se llevó a cabo el estudio. Los resultados del estudio indican que la reducción del comportamiento defensivo en la población ocurrió por el incremento de la frecuencia relativa de colonias con morfotipo y haplotipo europeo en la población como consecuencia del proceso de selección.

En 1996, el INIFAP inició un programa de mejoramiento genético apícola con el fin de generar líneas de abejas seleccionadas para alta producción de miel y bajo comportamiento defensivo. El programa de mejoramiento genético se desarrolló en el Estado de México y la población de aproximadamente 500 colonias de abejas bajo selección se formó con colonias del INIFAP y de apicultores cooperantes. Las características que se incluyeron como criterio de selección en el programa fueron: la producción de miel, el comportamiento defensivo, la longitud promedio del ala anterior de las abejas obreras de la colonia, el morfotipo de las colonias (europeo, híbrido o africanizado) y el haplotipo de las colonias (europeo o africano) ${ }^{(60)}$.

En este programa de mejoramiento genético se generaron tres líneas de abejas, cuyo desempeño para la producción de miel, comportamiento defensivo, longitud promedio del ala anterior de abejas obreras, distribución de morfotipos (europeo, híbrido y africanizado), distribución de haplotipos (europeo y africano) y la variabilidad genética del ADN mitocondrial ha sido evaluado y comparado con poblaciones de abejas europeas $\mathrm{y}$ africanizadas que no pertenecen a programas de mejoramiento genético en varios estudios $^{(61-65)}$. 
En lo que se refiere a la producción de miel, en dos estudios diferentes, las líneas de abejas mejoradas del INIFAP produjeron significativamente más miel que abejas que no pertenecían a un programa de mejoramiento genético ${ }^{(61,63)}$. En uno de los estudios, las líneas del INIFAP produjeron en promedio $34.5 \%$ más miel que abejas africanizadas no seleccionadas $^{(61)}$, mientras que en el otro estudio produjeron en promedio 27.5 y $40.3 \%$ más miel que abejas europeas y abejas africanizadas no seleccionadas respectivamente ${ }^{(63)}$.

En cuanto al comportamiento defensivo, medido por el número de aguijones que las abejas de una colonia depositan sobre una bandera de gamuza negra que se agita frente a la colmena por un tiempo determinado, se encontró que las líneas mejoradas fueron en promedio $57.4 \%$ menos defensivas que abejas africanizadas no seleccionadas, pero fueron $44.3 \%$ más defensivas que abejas europeas no seleccionadas ${ }^{(63)}$.

En relación a la longitud promedio del ala anterior de las abejas obreras de la colonia, en uno de los estudios en los que se evaluó esta característica, dos líneas de abejas del INIFAP tuvieron una longitud $(9.07$ y $9.06 \mathrm{~mm})$ mayor que la de abejas europeas $(9.03$ $\mathrm{mm}$ ) y africanizadas $(8.90 \mathrm{~mm})$, mientras que la otra línea del INIFAP tuvo una longitud del ala $(9.02 \mathrm{~mm})$ similar a la de abejas europeas no seleccionadas, y estos dos grupos tuvieron una longitud del ala mayor que la de abejas africanizadas no seleccionadas ${ }^{(63)}$. En el otro estudio, se encontró que las líneas de abejas mejoradas tuvieron una longitud del ala $(9.05,9.04$ y $9.03 \mathrm{~mm})$ mayor a la de abejas africanizadas no seleccionadas $(8.98$ $\mathrm{mm})^{(65)}$.

En cuanto a la distribución de los morfotipos de las colonias en la población, en los dos estudios en donde se evaluó esta característica, se encontró que en las poblaciones de las líneas seleccionadas las frecuencias de colonias de abejas con morfotipo europeo, híbrido $\mathrm{y}$ africanizado fueron significativamente diferentes a las de poblaciones de abejas no seleccionadas ${ }^{(62,65)}$. En un estudio se encontró que, en las poblaciones de abejas de las líneas seleccionadas, la frecuencia relativa promedio de colonias con morfotipo europeo fue 0.47 , con morfotipo híbrido 0.35 y africanizado 0.18 , mientras que en la población de colonias no seleccionadas la frecuencia relativa del morfotipo europeo fue 0.17 , de híbrido 0.43 y de africanizado fue $0.40^{(62)}$. En otro estudio, en la población de colonias de las tres líneas de abejas del INIFAP, las frecuencias relativas promedio de colonias con morfotipo europeo, híbrido y africanizado fueron $0.37,0.42$ y 0.21 respectivamente, mientras que en la población de abejas no seleccionadas fueron $0.17,0.43$ y $0.41^{(65)}$.

Para la distribución de los haplotipos, se encontró que en las poblaciones de las líneas seleccionadas, las frecuencias relativas promedio de colonias de abejas con haplotipo europeo y africano fueron significativamente diferentes a las de poblaciones de abejas no seleccionadas, la frecuencia del haplotipo europeo fue 0.93 y la de haplotipo africano fue 0.07 en la población de abejas del INIFAP, mientras que en la población no seleccionada fueron 0.34 y 0.66 respectivamente ${ }^{(62)}$. Finalmente, en cuanto a la variabilidad genética del ADN mitocondrial, estimada por el índice de Shannon, las líneas seleccionadas 
presentaron una menor variabilidad (IS=0.12) que las poblaciones no seleccionadas (IS=0.41), como consecuencia del proceso de selección al que han sido sometidas ${ }^{(64)}$.

Las líneas de abejas mejoradas se han utilizado para el desarrollo de proyectos en los que el INIFAP ha transferido el material genético mejorado a grupos de apicultores en el Estado de México, Hidalgo, Querétaro y Morelos, a través de la entrega de 10,000 celdas reales, 3,000 reinas de libre fecundación y 500 reinas inseminadas instrumentalmente para ser utilizadas como pie de cría, a partir de las cuales los apicultores han producido al menos 20,000 reinas de libre fecundación ${ }^{(66)}$.

\section{Resultados de los trabajos realizados para la conservación de germoplasma apícola europeo}

Con objeto de conservar material genético apícola de origen europeo, el INIFAP estableció en el 2004 un Banco de Germoplasma ${ }^{(67)}$ que está ubicado en el Centro Nacional de Investigación Disciplinaria en Fisiología y Mejoramiento Animal. El banco está formado por una población de 100 colonias de abejas que se manejan bajo un esquema de población cerrada a través del uso de la inseminación instrumental de abejas reinas $^{(68,69)}$, lo que permite mantener un aislamiento genético de la población al tener control total sobre los apareamientos, ya que sólo las reinas y zánganos del banco de germoplasma se utilizan al hacer los cruzamientos para producir las reinas para el reemplazo anual de reinas que se tiene que realizar en la población.

El germoplasma se encuentra caracterizado en términos morfométricos, moleculares y de comportamiento y cada año se hacen evaluaciones de las colonias de abejas para garantizar que el material genético que se conserva en el banco tenga características europeas. Se realizan análisis genéticos en donde se utilizan marcadores moleculares localizados en el ADN mitocondrial, que permiten clasificar el haplotipo de las abejas en europeo o africano ${ }^{(70)}$. Se realizan análisis morfométricos en donde se mide la longitud del ala anterior de las abejas, lo que permite clasificar a las colonias en europeas, híbridas o africanizadas ${ }^{(71)}$. Se evalúa el comportamiento defensivo de las colonias por medio de pruebas de apreciación en las que se mide qué tanto las abejas de una colonia corren sobre el panal, vuelan sobre la colmena, chocan y aguijonean al apicultor cuando se hace una revisión de rutina ${ }^{(60)} \mathrm{y}$, finalmente, también se evalúa la tendencia de las colonias a enjambrar y a evadir.

El banco de germoplasma apícola del INIFAP se ha conservado de esta forma por 17 generaciones, y cada generación corresponde a un ciclo apícola de un año. El material genético del banco se ha utilizado para el desarrollo de proyectos de investigación, así como para la generación, validación y transferencia de material genético mejorado ${ }^{(66)}$. 


\section{Resultados de estudios realizados sobre la genética de la resistencia de las abejas a la varroosis}

En México se realizaron estudios con el objetivo de determinar si existen diferencias en la susceptibilidad de las abejas africanizadas y europeas a la infestación del ácaro Varroa destructor $^{(72,73)}$. En el estudio desarrollado por Guzmán-Novoa et al ${ }^{(72)}$, se comparó la susceptibilidad de las abejas adultas y de la cría de abejas con genotipo africanizado, europeo y sus dos híbridos recíprocos a ser infestadas por Varroa destructor. Los resultados del estudio indican que para el caso de las abejas adultas, los genotipos africanizado y los dos híbridos fueron igual de susceptibles, pero fueron menos susceptibles a ser infestados que el genotipo europeo. En el caso de la cría, el genotipo africanizado fue el menos susceptible a ser infestado, seguido de los genotipos europeo y el híbrido de madre europea y padre africanizado, mientras que el híbrido de madre africanizada y padre europeo fue el más susceptible. En este mismo estudio se evaluó si estos genotipos tenían un efecto sobre la capacidad reproductiva del ácaro y se encontró que la capacidad reproductiva de Varroa destructor fue similar en los genotipos europeos y africanizado, y que su capacidad reproductiva fue mayor en los dos genotipos híbridos.

Guzmán-Nova et al $^{(73)}$ analizaron los resultados de varios estudios que se llevaron a cabo en México para conocer si la susceptibilidad a Varroa destructor era diferente entre las abejas africanizadas y europeas. Los resultados de estos trabajos indican que la susceptibilidad o resistencia al ácaro no depende del grupo genético de las abejas, ya que en los dos grupos existe variabilidad en la susceptibilidad de las colonias a ser infestadas por Varroa destructor. Asimismo, los resultados de los trabajos indicaron que el medio ambiente y la interacción entre el genotipo y el medio ambiente juegan un papel importante en los niveles de infestación que alcanza el ácaro en las colonias de abejas.

La resistencia de las colonias de abejas a Varroa destructor depende de la expresión de mecanismos de resistencia que tienen las abejas melíferas contra este ácaro; estos mecanismos incluyen el comportamiento de acicalamiento, el comportamiento higiénico, la atracción diferencial de la cría de obrera y de las abejas adultas al ácaro, un periodo de desarrollo de la cría más corto después de ser operculada y factores de las abejas que afectan la fertilidad y reproducción del ácaro ${ }^{(73-78)}$.

En México se realizó uno de los dos estudios que se han llevado a cabo para medir la contribución relativa de cada uno de estos mecanismos a la resistencia general de las colonias de abejas a Varroa destructor ${ }^{(75,79)}$. En el estudio que desarrollaron Arechavaleta-Velasco y Guzmán-Novoa ${ }^{(75)}$ se determinó que existe variabilidad de origen genético en la resistencia de las colonias de las abejas en México al crecimiento poblacional de Varroa destructor, y que la resistencia no está relacionada a una línea o grupo genético en particular. En este estudio se evaluó la contribución relativa de los comportamientos de acicalamiento e higiénico, de la atracción diferencial que ejerce la cría al ácaro y del efecto que tiene la cría sobre la capacidad reproductiva de Varroa 
destructor, a la resistencia al crecimiento poblacional del ácaro en las colonias de abejas. Los resultados de este trabajo indican que el comportamiento de acicalamiento es el principal mecanismo de resistencia contra el crecimiento poblacional de Varroa destructor en las colonias. Asimismo, se encontró que las colonias susceptibles y resistentes presentaron diferencias en la expresión del comportamiento higiénico pero su contribución en la resistencia al ácaro no fue clara, mientras que en el estudio desarrollado por Harbo y Harris ${ }^{(79)}$ se identificó al comportamiento higiénico específico contra Varroa destructor como el principal mecanismo de resistencia.

Arechavaleta-Velasco et $a l^{(80)}$ desarrollaron un estudio para identificar las regiones dentro del genoma de la abeja que regulan la expresión del comportamiento de acicalamiento de las abejas. En este trabajo se identificó un locus de rasgos cuantitativos (QTL), denominado groom-1, en el cromosoma 5 del genoma de la abeja para el comportamiento de acicalamiento. Dentro de la región que corresponde al intervalo de confianza al $95 \%$ para la ubicación del QTL se identificaron 27 genes reportados en el Honeybee genome database, uno de estos genes es neurexin-1 (AmNrxl). Los ortólogos de este gen están relacionados con el autismo y la esquizofrenia en humanos, con la formación de sinapsis y el aprendizaje por asociación en Drosophila y Aplysia, y con el comportamiento de acicalamiento en ratones. En ratones knockout para neurexin-1-alpha se incrementa el comportamiento de auto acicalamiento en comparación con otros ratones. El hecho de que neurexin-1 influya en el comportamiento de acicalamiento tanto en mamíferos como en abejas, es evidencia del efecto de este gen sobre la expresión de la característica. El efecto del QTL groom-1 y del gen neurexin-1 (AmNrxl) sobre la expresión del comportamiento de acicalamiento se confirmaron posteriormente en una población de abejas diferente a la población en donde se identificó por primera vez el efecto del QTL y el gen ${ }^{(81)}$.

Para identificar regiones del genoma que regulan la expresión del comportamiento higiénico se realizó un estudio ${ }^{(82)}$ en el que se detectaron siete BTL asociados a la expresión de esta característica a nivel de abejas individuales; el número de BTL que se identificó en este estudio fue similar al número de QTL detectados en el estudio desarrollado por Lapidge et $a l^{(83)}$ para identificar regiones genómicas para esta característica a nivel del fenotipo de toda la colonia de abejas.

\section{Impactos}

Los trabajos que se han desarrollado en el INIFAP relacionados con la genética del comportamiento defensivo de las abejas y de los mecanismos de resistencia de las abejas a Varroa destructor han tenido un impacto científico importante a nivel mundial. Estos trabajos han permitido generar conocimientos de frontera sobre los mecanismos genéticos, genómicos y epigenéticos que regulan la expresión del comportamiento defensivo de las colonias, de los comportamientos de guardia y aguijoneo de abejas individuales, del comportamiento de acicalamiento y del comportamiento higiénico. 
En materia de mejoramiento genético para reducir el comportamiento defensivo de las colonias de abejas, los trabajos que se han realizado han tenido un impacto científico y tecnológico importante, porque han permitido generar métodos para la evaluación, la selección y el mejoramiento genético de esta característica. Utilizando estos conocimientos se generaron líneas de abejas de bajo comportamiento defensivo a partir de las cuales se han producido 500 reinas pie de cría y al menos 33,000 reinas de libre fecundación que se han transferido a productores, lo que tiene un impacto social y productivo importante.

Los trabajos desarrollados por el INIFAP para establecer un Banco de Germoplasma Apícola tienen un impacto ecológico y tecnológico importante, ya que este banco es el único núcleo de conservación de germoplasma de origen europeo que existe en México, y las abejas que se mantienen en el banco son un recurso genético importante para la apicultura del país que se debe conservar, ya que existe un alto riesgo de perder el germoplasma de origen europeo debido a los altos niveles de africanización que existen en las poblaciones de abejas de México.

\section{Literatura citada:}

1. FAO. Organización de las Naciones Unidas para la Alimentación y la Agricultura. Food and agriculture data. 2020. http://www.fao.org/faostat/en/\#data/QA Consultado 1 Ago, 2020.

2. SIAP. Servicio de Información Agroalimentaria y Pesquera. Anuario Estadístico de la Producción Ganadera. México. 2010-2019. https://nube.siap.gob.mx/cierre_pecuario/CConsultado 1 Ago, 2020.

3. Vélez IA, Espinosa GJ, Gutiérrez RA, Arechavaleta-Velasco ME. Tipología y caracterización de apicultores del estado de Morelos, México. Rev Méx Cienc Pecu 2016;7(4):507-524.

4. Guzmán-Novoa E. La apicultura en México y Centroamérica. Congreso Iberolatinoamericano Apícola. Mercede, Uruguay. UNA;1996:14-17.

5. García GL, Meza RE. Oportunidades y obstáculos para el desarrollo de la apicultura en Nayarit.http://www.eumed.net Consultado 25 Nov, 2012.

6. Kerr WE. The history of the introduction of Africanized bees to Brazil. S Afr Bee J 1967;39:3-5.

7. Winston ML. The biology and management of the Africanized honey bees. Annu Rev Entomol 1992;37:173-193.

8. Moffett JO, Maki DL, Andre T, Fierro MM. The Africanized bees in Chiapas, Mexico. Am Bee J 1987;127:520-571. 
9. Sudgen EA, Williams KR. October 15: the day the bee arrived. Glean Bee Cult 1991;119:18-21.

10. Winston ML. The Biology of the honey bee. Harvard University Press. EUA 1987.

11. Guzmán-Novoa E, Page RE. The impact of Africanized bees on Mexican beekeeping. Ann Bee J 1994;124(2):101-106.

12. Collins AM, Rinderer TE, Harbo J. Colony defense by Africanized and European honey bees. Science 1982;218:72-74.

13. Otis GW. Population biology of the Africanized honey bee. In: Jaisson P, editor. Social insects in the tropics. 1982;1:209-219.

14. Hunt G, Guzmán-Novoa E, Fondrk MK, Page RE. Quantitative trait loci for honey bee stinging behavior and body size. Genetics 1998;149:1203-1213.

15. Guzmán-Novoa, Uribe-Rubio. Honey production by european, africanized and hybrid honey bee (Apis mellifera) colonies in Mexico. Am Bee J 2004;144:318-320.

16. Domínguez-Ayala R, Moo-Valle H, May-Itza W, Medina-Peralta S, Quezada-Euán J. Stock composition of northern neotropical honey bees: mitotype and morphotype diversity in Mexico (Hymenoptera: Apidae). Apodologie 2016;47:642-652.

17. Clarke K, Oldroyd B, Quezada-Euán J, Rinderer T. Origin of honeybees (Apis mellifera $\mathrm{L}$.) from the Yucatán peninsula inferred from mitochondrial DNA analysis. Mol Ecol 2001;10:1347-1355.

18. Arechavaleta VM, Robles RC, Espinosa SA. Caracterización molecular y morfométrica de las poblaciones de colonias de abejas del Estado de México. Reunión Nacional de Investigación Pecuaria. Mérida, Yucatán. 2008:176.

19. Arechavaleta VM, Vázquez PS, Ramírez RF, Camacho RC, Robles RC, Amaro GR. Distribución de los morfotipos europeo, africanizado e híbrido en poblaciones de colonias de abejas de Morelos. Reunión Nacional de Investigación Pecuaria. Veracruz. 2013:456.

20. Arechavaleta-Velasco ME, Guzmán-Novoa E. Producción de miel de colonias de abejas (Apis mellifera L.) tratadas y no tratadas con un acaricida contra Varroa jacobsoni Oudemans en Valle de Bravo, Estado de México. Rev Vet Méx 2000;31:381-384.

21. Medina-Flores CA, Guzmán-Novoa E, Aréchiga-Flores CF, Aguilera-Soto JI, Gutiérrez-Piña FJ. Efecto del nivel de infestación de Varroa destructor sobre la producción de miel de colonias de Apis mellifera en el altiplano semiárido de México. Rev Mex Cienc Pecu 2011;2(3):313-317. 
22. Medina-Flores CA, Esquivel-Marín NH, López-Carlos M, Medina-Cuellar SE, Aguilera-Soto JI. Estimación de la pérdida de colonias de abejas melíferas en el altiplano y el norte de México. Exosist Recur Agropec 2018;5(14):365-371.

23. Arechavaleta VM, Velez IA, Espinosa GJ, Amaro GR, Alcalá EB, Vázquez PS, Ramírez RF. Presencia del síndrome del colapso de las colonias de abejas (CCD) en México. Reunión Nacional de Investigación Pecuaria. Mérida, Yucatán. 2014:82.

24. Genersch E, Von Der OH, Kaatz H, Schroeder A, Otten C, Büchler R, et al. The German bee monitoring project: a long term study to understand periodically high winter losses of honey bee colonies. Apidologie 2010;41(3):332-352.

25. Currie RW, Pernal SF, Guzmán-Novoa E. Honey bee colony losses in Canada. J Apic Res 2010;49:104-106.

26. Dahle B. The role of Varroa destructor for honey bee colony losses in Norway. J Apic Res 2010;49:124-125.

27. Peterson M, Gray A, Teale A. Colony losses in Scotland in 2004-2006 from a sample survey. J Apic Res 2010;48:145-146.

28. Rodríguez-Dehaibes SR, Otero-Colina G, Villanueva-Jiménez, Corcuera MRP, Chávez VC, Lagunas ZR. Resistencia de Varroa destructor a los plaguicidas usados para su control en las regiones Golfo y Centro-Altiplano, México. Congreso Internacional de Actualización Apícola; 2007 mayo 16-18; Boca del Río, Veracruz. 2007:40-44.

29. Arechavaleta VM, Torres NG, Robles RC, Correa BA. Identificación de poblaciones de Varroa destructor resistentes al fluvalinato en colonias de abejas en el Estado de México. Cong Int Actualiz Apícola. Boca del Río, Veracruz. 2007:113-116.

30. Slabezki Y, Gal H, Lensky Y. The effect of fluvalinate application in bee colonies on population levels of Varroa jacobsoni and honey bees (Apis mellifera L.) and on residues in honey and wax. Bee Sci 1991;1(4):189-195.

31. Lodesani M, Pellacani A, Bergomi S, Carpana E, Rabitti T, Lasagni P. Residue determination for some products used against Varroa infestation in bees. Apidologie 1992;23(3):257-272.

32. Wallner K. Varroacides and their residues in bee products. Apidologie 1999;30:235248.

33. Mathieu L, Faucon JP. Changes in the response time for Varroa jacobsoni exposed to amitraz. J Apicult Res 2010;39(3-4):155-158.

34. Pettis JS, Collins AM, Wilbanks R, Feldlaufer MF. Effects of coumaphos on queen rearing in the honey bee, Apis mellifera. Apidologie 2004;35(6):605-610. 
35. Guzmán NE, Emsen B, Gashout H, Rodríguez MF, Correa BA. Eficacia de productos orgánicos y de diferentes métodos de aplicación en el control del ácaro Varroa destructor y su inocuidad en las abejas melíferas. $14^{\circ}$ Congreso Internacional de Actualización Apícola; 2007 mayo 16-18; Boca del Río, Veracruz. 2007:45-51.

36. Chihu AD, Rojas AM, Rodríguez DS. Presencia en Veracruz, México del ácaro Varroa jacobsoni, causante de la varroasis de la abeja melífera (Apis mellifera L.). Tec Pecu Méx 1992;30(2):133-135.

37. Medina-Flores CA, Guzmán-Novoa E, Hamiduzzaman MM, Aréchiga-Flores CF, López-Carlos MA. Africanized honey bees (Apis mellifera) have low infestation levels of the mite Varroa destructor in different ecological regions in Mexico. Genet Mol Res 2014;13(3):7282-7293.

38. Vázquez PS, Arechavaleta VME, Ramírez RFJ, Amaro GR, Robles RC. Prevalencia y niveles de infestación de Varroa destructor en colonias de abejas del Estado de Morelos. Reunión Nacional de Investigación Pecuaria. Veracruz. 2013:66.

39. Tapia-González JM, Alcazar-Oceguera G, Macías-Macías JO, Contreras-Escareño F, Tapia-Rivera JC, Petukhova T, Guzmán-Novoa E. Varroosis en las abejas melíferas en diferentes condiciones ambientales y regiones de Jalisco, México. Ecosist Recur Agropec 2019;6(17):243-251.

40. Martínez PJ, Medina ML, Catzin VG. Frecuencia de Varroa destructor, Nosema apis y Acarapis woodi en colonias manejadas y enjambres silvestres de abejas (Apis mellifera) en Mérida, Yucatán, México. Rev Mex Cienc Pecu 2011;2(1):25-38.

41. Guzmán-Novoa E, Page RE. Backcrossing Africanized honey bee queens to European drones reduces colony defensive behavior. Ann Entomol Soc Am 1993;86:352-355.

42. Guzmán-Novoa E, Page RE. Genetic dominance and worker interactions affect honey bee colony defense. Behav Ecol 1994;5:91-97.

43. Guzmán-Novoa E, Hunt GJ, Uribe JL, Smith C, Arechavaleta-Velasco ME. Confirmation of QTL effects and evidence of genetic dominance of honeybee defensive behavior: results of colony and individual behavioral assays. Behav Genet 2002;32:95-105.

44. Arechavaleta-Velasco ME, Hunt GJ, Emore C. Quantitative trait loci that influence the expression of guarding and stinging behaviors of individual honey bees. Behav Genet 2003;33:357-364.

45. Moore AJ, Brees MD. Morr MJ. The guard honey bee-ontogeny and behavioral variability of workers performing a specialized task. Anim Behav 1987;35:11591167.

46. Breed DM, Robinson GE, Page RE. Division of labor during honey-bee colony defense. Behav Ecol Sociobiol 1990;27:395-401. 
47. Breed DM, Guzmán-Novoa E, Hunt GJ. Defensive behavior of honey bees: organization, genetics, and comparisons with other bees. Annu Rev Entomol 2004;49:271-298.

48. Guzmán-Novoa E, Hunt GJ, Uribe-Rubio JL, Prieto-Merlos D. Genotypic effects of honey bee (Apis mellifera) defensive behavior: results of colony and individual behavioral assays. Behav Genet 2004;32:95-102.

49. Uribe-Rubio JL, Guzmán-Novoa E, Vasquez-Pelaez CG, Hunt GJ. Genotype task specialization, and nest environment influence the stinging response thresholds of individual Africanized and European honeybees to electrical stimulation. Behav Genet 2008;38:93-100.

50. Hunt GJ, Guzmán-Novoa E, Uribe-Rubio JL, Prieto-Merlos D. Genotype environment interactions in honeybee guarding behavior. Anim Behav 2003;66:459467.

51.Arechavaleta-Velasco ME, Hunt GJ. Genotypic variation in the expression of guarding behavior and the role of guards in the defensive response of honey bee colonies. Apidologie 2003;34:439-447.

52. Shorter JR, Arechavaleta-Velasco ME, Robles- Ríos C, Hunt GJ. A genetic analysis of the stinging and guarding behaviors of the honeybee. Behav Genet 2012;42:663674.

53. Arechavaleta-Velasco ME, Hunt GJ. Binary trait loci that influence honey bee (Hymenoptera: Apidae) guarding behavior. Ann Entomol Soc Am 2004;97:177-183.

54. Guzmán-Novoa E, Hunt GJ, Page RE, Uribe-Rubio JL, Prieto-Merlos D, BecerraGuzmán F. Paternal effects of the defensive behavior of honeybees. J Hered 2005;96:376-380.

55. Queller DC. Theory of genomic imprinting conflict in social insects. BMC Evol Biol 2003;15(3):1-23.

56. Ferguson-Smith AC. Genomic imprinting: the emergence of an epigenetic paradigm. Nature Reviews. Genetics 2011;12:565-575.

57. Kocher SD, Tsuruda JM, Gibson JD, Emore CM, Arechavaleta-Velasco ME, Queller $\mathrm{D}$, et al. A search for parent of origin effects on honey bee gene expression. G3 2015;5:1657-1662.

58. Gibson JD, Arechavaleta-Velasco ME, Tsuruda JM, Hunt GJ. Biased allele expression and aggression in hybrid honeybees may be influenced by inappropriate nuclear-cytoplasmic signaling. Front Genet 2015;6:343.

59. Guzmán-Novoa E, Page Jr RE. Selective breeding of honey bees (Hymenoptera: Apidae) in africanized areas. J Ecom Entomol 1999;92(3):521-525. 
60. Arechavaleta-Velasco ME. Mejoramiento genético de las abejas melíferas. Folleto Técnico N 17 INIFAP-CENID Fisiología y Mejoramiento Animal. 2012.

61. Guzmán NE, Uribe RJL, Prieto MD. Producción de miel de tres estirpes de abejas seleccionadas en el altiplano mexicano. Reunión Nacional de Investigación Pecuaria. CDMX. 2003:227.

62. Arechavaleta VME, Robles RCA, Uribe RJL, Guzmán NE. Distribución de mitotipos y morfotipos en poblaciones de colonias de abejas melíferas seleccionadas y no seleccionadas. Reunión Nacional de Investigación Pecuaria. Veracruz. 2006:199.

63. Arechavaleta VME, Robles RCA, García FF, Noriega G, Uribe RJL. Producción de miel, comportamiento defensivo y longitud promedio del ala anterior de colonias de abejas desarrolladas bajo dos métodos de selección. Reunión Nacional de Investigación Pecuaria. Sinaloa. 2007:205.

64. García FF, Arechavaleta VME, Robles RCA. Variabilidad genética del ADN mitocondrial en poblaciones de abejas melíferas seleccionadas y no seleccionadas. Reunión Nacional de Investigación Pecuaria. Saltillo, Coahuila. 2009:98.

65. Alcalá EKI, Arechavaleta VME, Robles RCA. Niveles de africanización en poblaciones de colonias de abejas desarrolladas bajo dos métodos de selección en zonas africanizadas. Reunión Nacional de Investigación Pecuaria. Campeche, Campeche. 2010:208.

66. Arechavaleta VM, Guzmán-Novoa E, Uribe RJ, García FC, Ramírez RF. Mejoramiento genético para el manejo de la africanización de colonias de abejas melíferas. In: Cruz Cruz E, Reyes-Muro L compiladores. Aportaciones del INIFAP al campo mexicano en 35 años. Libro Técnico N 1 INIFAP 2020.

67. GRIN. Animal Germplasm Resources Information Network. Centro Nacional de Recursos Genéticos-INIFAP. https://agrin.ars.usda.gov/database_collaboration_page_dev Consultado 17 Mar, 2021.

68. Page Jr RE, Laidlaw Jr HH. Closed population honeybee breeding. Bee world 1985;66(2):63-72.

69. Cobey S, Lawrence T. Commercial application and practical use of the Page-Laidlaw closed population breeding program. Am Bee J 1988;128(5):341-344.

70. Nielsen DI, Ebert PR, Page RE, Hunt GJ, Guzmán-Novoa E. Improved polymerase chain reaction based mitochondrial genotype assay for identification of the africanized honey bee (Hymenoptera: Apidae). Ann Entomol Soc Am 2000;93:1-6.

71. Sylvester HA, Rinderer TE. Fast africanized bee identification system. Ame Bee J 1987;127(7):511-516. 
72. Guzmán-Novoa E, Sánchez A, Page Jr RE, García T. Susceptibility of European and Africanized honeybees (Apis mellifera L.) and their hybrids to Varroa jacobsoni Oud. Apidologie 1996;27(2):93-103.

73. Guzmán-Novoa E, Vandame R, Arechavaleta-Velasco ME. Susceptibility of European and Africanized honeybees (Apis mellifera L.) to Varroa jacobsoni Oud. in México. Apidologie 1999;30:173-182.

74. Moosbeckhofer R. Beobachtungen sum Auftreten beschädigter Varroamilben im natürlichen Totenfall bei Völkern von Apis mellifera carninca. Apidologie 1992;23:523-531.

75. Arechavaleta-Velasco ME, Guzmán-Novoa E. Relative effect of four characteristics that restrain the population growth of the mite Varroa destructor in honey bee (Apis mellifera) colonies. Apidologie 2001;32:157-174.

76. Mondragón L, Spivak M, Vandame R. A multifactorial study of the resistance of honeybees Apis mellifera to the mite Varroa destructor over one year in Mexico. Apidologie 2005;36(3):345-358.

77. Ibrahim A, Spivak M. The relationship between hygienic behavior and suppression of mite reproduction as honey bee (Apis mellifera) mechanisms of resistance to Varroa destructor. Apidologie 2006;37(1):31-40.

78. Ibrahim A, Reuter GS, Spivak M. Field trial of honey bee colonies bred for mechanisms of resistance against Varroa destructor. Apidologie 2007;38(1):67-76.

79. Harbo JR, Harris JW. Selecting honey bees for resistance to Varroa jacobsoni. Apidologie 1999;30(2-3):183-196.

80. Arechavaleta-Velasco ME, Alcala-Escamilla K, Robles-Rios C, Tsuruda JM, Hunt GJ. Fine-scale linkage mapping reveals a small set of candidate genes Influencing honey bee grooming behavior in response to varroa mites. PLOS ONE 2012;7(11):e47269.

81. Hamiduzzaman M, Emsen B, Hunt GJ, Subramanyam S, Williams CE, Tsuruda JM, Guzmán-Novoa E. Differential gene expression associated with honey bee grooming behavior in response to Varroa mites. Behav Genet 2017;47(3):335-344.

82. Arechavaleta-Velasco ME, Hunt GJ, Spivak M, Camacho-Rea C. Loci de rasgos binarios que influyen en la expresión del comportamiento higiénico de las abejas melíferas. Rev Mex Cienc Pecu 2011;2(3):283-298.

83. Lapidge KL, Oldroyd BP, Spivak M. Seven suggestive quantitative trait loci influence hygienic behavior of honey bees. Naturwissenschaften 2002;89:565-568. 